\title{
Perfil dos atendimentos realizados pelo Serviço de Atendimento Móvel de Urgência no município de Colombo (PR)
}

\author{
Profile of care provided by the Mobile Emergency Care service of the city of \\ Colombo-PR
}

Perfil de asistencia realizado por el Servicio de Emergencia Móvil en el municipio de Colombo-PR

\begin{abstract}
Jéssica Sanches Silva ${ }^{1}$, Thais Lazaroto Roberto Cordeiro ${ }^{2}$, Maria de Lourdes Neris Carvalho $^{3}$, Itacira Terezinha dos Santos Silva ${ }^{4}$

\footnotetext{
${ }^{1}$ Enfermeira. Especialista em Urgência e Emergência. Faculdade Inspirar. Curitiba - Paraná

${ }^{2}$ Mestre. Faculdades Pequeno Príncipe - FPP. Curitiba - Paraná
}

${ }^{3}$ Enfermeira. Especialista em Urgência e Emergência. Faculdade Inspirar. Curitiba - Paraná

${ }^{4}$ Enfermeira. Especialista em Urgência e Emergência. Faculdade Inspirar. Curitiba - Paraná
\end{abstract}

\section{RESUMO}

Objetivo: Descrever o perfil dos atendimentos realizados pelo Serviço de Atendimento Móvel de Urgência (SAMU) do município de Colombo (PR). Método: Estudo descritivo de abordagem quantitativa, utilizando as informações contidas nas fichas de atendimentos do SAMU do município de Colombo (PR) para a coleta dos dados no período de dezembro de 2016 a agosto de 2017. Resultados: Foram analisados 2.784 fichas e os resultados mostram predominância do

Autor de Correspondência:

*Jéssica Sanches Silva. E-mail: jessicasanchess@hotmail.com 
sexo masculino com 1.441 (51,76\%), faixa etária de 19-59 anos com 1.531 (54,99\%), ocorrências de perfil clínico com 1.715 (61,60\%) e, tendo como destino final a UPA do município 1.377 casos (63,75\%). Conclusões: O estudo contribui na quantificação de informações de atendimento do SAMU realizados no município de Colombo, a fim de qualificar a organização das demandas de atendimento pela rede de Urgência e Emergência no município.

Palavras-chave: Perfil de Saúde. Emergências. Serviços Médicos de Emergência. Unidades Móveis de Saúde.

\begin{abstract}
Objective: To describe the profile of care provided by the Mobile Emergency Care Service (SAMU) of the city of Colombo-PR. Method: Descriptive study of quantitative approach, using the information contained in the SAMU service records of the city of Colombo-PR for data collection from December 2016 to August 2017. Results: A total of 2784 records were analysed and the results show a predominance of males with 1441 patients (51.76\%), age group of 19-59 with 1531 cases (54.99\%), occurrences of clinical profile with 1715 (61.60\%), and with UPA of the municipality as final destination with 1377 cases (63.75\%). Conclusion: The study contributes to the quantification of information about care by SAMU carried out in the city of Colombo, in order to qualify the organization of care demands by the Urgency and Emergency network in the Municipality.
\end{abstract}

Keywords: Health Profile. Emergencies. Emergency Medical Services. Mobile Health Units.

\title{
RESUMEN
}

Objetivo: Describir el perfil de la atención realizada del Servicio de Emergencia Móvil (SAMU) en la ciudad de Colombo-PR. Método: un estudio descriptivo con un enfoque cuantitativo, que utiliza la información contenida en los archivos de asistencia de SAMU en la ciudad de Colombo-PR para recopilar los datos desde diciembre de 2016 hasta agosto de 2017. Resultados: se analizaron 2784 registros y los resultados muestran un predominio en varones con 1441 (51.76\%), en el grupo de edad de 19-59 años con 1531 (54.99\%), ocurrencias de perfil clínico con 1715 (61.60 \%) y con destino final a los casos UPA 1377 de la ciudad (63.75\%). Conclusión: El estudio contribuye a la cuantificación de la información de atención SAMU realizada en la ciudad de Colombo, para calificar la organización de las demandas de atención por parte de la red de Urgencias y Emergencias en la Ciudad.

Palabras clave: Perfil de Salud. Urgencias Médicas. Servicios Médicos de Urgencia. Unidades Móviles de Salud. 


\section{INTRODUÇÃO}

Os indivíduos sofrem situações que desestabilizam suas condições vitais, por isso precisam de uma assistência precoce e eficaz em situações de agravos clínicos, e uma das principais procuras destes usuários são os serviços de atendimento préhospitalar móvel ${ }^{1 .}$

Em 2003 foram instituídas no Brasil duas portarias que impulsionaram o Serviço de Atendimento Préhospitalar (APH): Portaria no 1.863 GM, que lança a Política Nacional de Atenção às Urgências e a Portaria no 1.864 GM que implementa o Serviço de Atendimento Móvel de Urgência $(S A M U)^{2}$. Este serviço móvel, além de ser o responsável por realizar atendimentos de urgência e emergência em locais como via pública, domicílios e estabelecimentos de saúde, também vêm sendo considerado como um identificador de dados epidemiológicos da população solicitante, podendo-se identificar os principais problemas de saúde e necessidades de um determinado local ${ }^{3}$.

O SAMU do município de Colombo foi fundado em dezembro de 2016, com sua base localizada ao lado da UPA Alto Maracanã, possuindo duas ambulâncias de Suporte Básico de Vida (SBV). Atualmente, o serviço atende a população deste município, cujo número de habitantes era 212.967 pessoas, segundo o último censo do IBGE em 2010. A estimativa para o ano de 2019 é de 243.726 habitantes.

No contexto, o sistema de saúde às urgências e emergências vem apresentando uma demanda elevada nos atendimentos pela população. Este fato está relacionado não somente por utilizarem o serviço de maneira incorreta, como também pela dificuldade que se deparam no acesso aos atendimentos na rede de atenção primária ${ }^{4}$

Algumas pesquisas mostraram que a utilização repetida dos serviços de urgência pelos usuários com características clínicas acontece por agudizações de doenças crônicas ${ }^{4}$. Em um estudo realizado em 2018, este apontamento é mostrado a partir do resultado dos dados do município de Ipatinga (MG), onde foram analisados 1020 boletins de ocorrências do SAMU sendo verificada a prevalência de ocorrências clínicas com 447 casos (43,82\%), sendo o quadro de dispneia com o maior indicador clínico 119 (11,67\%)

Partindo do pressuposto da alta demanda do serviço e procurando viabilizar a melhor implementação e eficácia do mesmo, propomos a seguinte pergunta de pesquisa: Qual o perfil dos atendimentos realizados pelo SAMU no município de Colombo (PR), no período de dezembro de 2016 a agosto de 2017?

Como objetivo, o presente estudo propôs-se a descrever o perfil dos atendimentos realizados pelo Serviço de Atendimento Móvel de Urgência (SAMU) do município de Colombo (PR).

\section{MÉTODO}

Trata-se de uma pesquisa descritiva de abordagem quantitativa, com finalidade de apontar o perfil dos atendimentos realizados pelo SAMU no município de Colombo (PR).

Os dados foram coletados das fichas dos atendimentos utilizadas nas ocorrências pelos profissionais do SAMU, referentes ao período de dezembro de 2016 a agosto de 2017. As fichas, elaboradas pelo município de Colombo, se encontram arquivadas na unidade de pronto atendimento e contém informações como dados pessoais do paciente, natureza da chamada, avaliação e exame clínico e encaminhamentos.

Para obter a coleta de dados, foi aberto previamente um protocolo na Prefeitura do município (Processo no 21.445/2018) para solicitar a autorização do estudo. Conforme orientações dadas neste primeiro processo, foi aberto um novo protocolo (Processo no 3629/2019), sendo realizados os requisitos recomendados para deferimento da pesquisa. 
Neste estudo, foram selecionados os seguintes dados para análise: demográficos (sexo e faixa etária), tipo de ocorrência (clínico, trauma, psiquiátrico, obstétrico e transferência), origem do atendimento, destino do paciente e encaminhamento (desfecho) do atendimento. Foram incluídas todas as fichas do período de dezembro de 2016 a agosto de 2017, sendo excluídas do banco de dados 398 fichas, por apresentarem seus relatórios rasurados e/ou ilegíveis. Após a coleta, os dados foram organizados e agrupados em uma planilha do Excel $^{\circ}$, distribuídos e apresentados em forma de tabela para análise de forma descritiva.
A pesquisa respeitou os preceitos éticos da Resolução n 466/12, do Conselho Nacional de Saúde, e foi aprovado pelo Comitê de Ética em Pesquisa da Faculdade Inspirar, sob o parecer n⿳⺈ 3.344.384.

\section{RESULTADOS}

Os dados foram coletados das fichas dos atendimentos do SAMU do município de Colombo $(\mathrm{PR})$, e correspondem ao número de atendimentos $(\mathrm{n}=2784)$ no período compreendido entre os meses de dezembro de 2016 a agosto de 2017, apresentados por meio da distribuição e análise categórica.

Tabela 1. Tabela para distribuição de faixa etária, sexo, tipos de ocorrência, destino e tipos de fechamento realizados entre os meses de dezembro de 2016 a agosto de 2017

\begin{tabular}{|c|c|c|}
\hline \multicolumn{2}{|c|}{ Variável } & \multirow{2}{*}{$\frac{\text { Total }(\mathbf{F}-\mathbf{\%})}{(\mathrm{n}=2784)}$} \\
\hline & & \\
\hline \multirow{5}{*}{$\begin{array}{l}\text { Faixa etária } \\
\text { dos usuários }\end{array}$} & 0 a 11 anos & $150-5,4 \%$ \\
\hline & 12 a 18 anos & $155-5,6 \%$ \\
\hline & 19 a 59 anos & $1531-55,0 \%$ \\
\hline & 60 anos ou mais & $795-28,6 \%$ \\
\hline & Idade não informada & $153-5,5 \%$ \\
\hline \multirow{3}{*}{$\begin{array}{l}\text { Sexo dos } \\
\text { usuários }\end{array}$} & Feminino & $1226-44,0 \%$ \\
\hline & Masculino & $1441-51,8 \%$ \\
\hline & Não informado & $117-4,2 \%$ \\
\hline \multirow{5}{*}{$\begin{array}{l}\text { Tipos de } \\
\text { ocorrência }\end{array}$} & Clínico & $1715-61,6 \%$ \\
\hline & Trauma & $581-20,9 \%$ \\
\hline & Psiquiátrico & $153-5,5 \%$ \\
\hline & Obstetrício & $117-4,2 \%$ \\
\hline & Transferências & $218-7,8 \%$ \\
\hline \multirow{5}{*}{$\begin{array}{l}\text { Destino do } \\
\text { transporte }\end{array}$} & Hospital de referência & $783-28,1 \%$ \\
\hline & UPA do município & $1377-49,5 \%$ \\
\hline & Local de origem & $275-9,9 \%$ \\
\hline & Outros & $122-4,4 \%$ \\
\hline & Não informado & $227-8,2 \%$ \\
\hline \multirow{4}{*}{$\begin{array}{l}\text { Tipo de } \\
\text { fechamento }\end{array}$} & Liberado após atendimento & $39-1,4 \%$ \\
\hline & Recusa atendimento & $229-8,2 \%$ \\
\hline & Transporte & $2129-76,5 \%$ \\
\hline & Não informado & $387-13,9 \%$ \\
\hline
\end{tabular}

Fonte: As autoras (Banco de dados do SAMU de Colombo - PR) 
A faixa etária dos usuários do SAMU em ordem decrescente pelo número de atendimentos é de 1531 casos $(54,99 \%)$ para a faixa entre 19 e 59 anos. Para a faixa de maiores de 60 anos foram realizados 795 $(28,56 \%)$ atendimentos. As crianças e adolescentes somam 305 (10,96\%), sendo distribuídos em 150 casos (5,39\%) para menores de 11 anos e 155 casos para adolescentes $(5,57 \%)$ de 12 a 18 anos. O número de casos encontrados sem registro de faixa etária foi de $153(5,50 \%)$.

Quanto ao sexo dos usuários, 1441 casos (51,76\%) são de atendimentos masculinos e $1226(44,04 \%)$ são de atendimentos femininos. Para esta categoria, 117 casos $(4,20 \%)$ não registraram o sexo dos usuários.

Os tipos de ocorrência no total do período apresentaram (em ordem decrescente), 1715 (61,60\%) clínicos, 581 (20,87\%) de trauma, 218 (7,83\%) de transferências, 153 (5,50\%) psiquiátricos e 117 (4,20\%) obstetrícios. Todas as fichas analisadas, 2784 (100\%), informaram o tipo de ocorrência.

O serviço de atendimento móvel teve como destino, em 1377 casos (63,75\%) na UPA do município, em 783 $(28,13 \%)$ os hospitais de referência, em 275 (9,88\%) permaneceram nos seus locais de origem, em 122 $(4,38 \%)$ destinaram a outros locais. Em 227 casos $(8,15 \%)$ analisados, não houve registro do local de destino.

No tocante aos encaminhamentos dados aos atendimentos do SAMU, apresentam-se 2129 casos $(76,47 \%)$ nos quais o desfecho foi o transporte, 387 (13,90\%) obtiveram liberação após o atendimento, 229 $(8,23 \%)$ recusa de atendimento e $39(1,40 \%)$ desfechos não foram devidamente informados.

\section{DISCUSSÃo}

A saúde pública nos últimos anos vem apresentando crescente demanda no serviço de urgência, devido aos usuários não iniciarem seus cuidados de prevenção na Atenção Primária à Saúde, principal porta de entrada do sistema. Dentre estes usuários, um estudo aponta um número significativo de usuários do sexo masculino em uma região metropolitana de Natal (RN), com $248(64,6 \%)$ dos casos. Na presente pesquisa realizada no SAMU de Colombo também houve predominância do sexo masculino, com 1441 $(51,76 \%)$ dos atendimentos ${ }^{1 .}$

No ano de 2018, pesquisadores afirmam que a procura por serviços de saúde no âmbito de prevenção e promoção é maior por mulheres do que os homens. Estes, por muitas vezes, deixam com que as agudizações de doenças crônicas ou doenças agudas se instalem para serem tratadas diretamente nos serviços de alta complexidade ${ }^{6}$. Outro estudo associa também esta falta de prevenção a um maior número de homens envolvidos em acidentes de trânsito, relacionados aos comportamentos sociais e culturais do grupo, como uso de álcool e imprudências no trânsito ${ }^{7}$.

Seguindo neste pensamento das comorbidades não tratadas, associa-se este fator para uma predominância de perfil clínico atendidos pelos serviços de urgência. Através de uma análise por meio de revisão integrativa, pode-se perceber que as características mais frequentes dos usuários foram os problemas respiratórios, neurológicos, psiquiátricos, circulatórios, endócrinos e gastrointestinais ${ }^{4}$.

No município de Colombo, os dados estatísticos mostraram que o quadro clínico foi o tipo de ocorrência mais solicitado pela população, com 1715 (61,60\%) casos. Pesquisas realizadas no município de Catanduva, Porto Alegre e no norte de Minas Gerais evidenciam resultados semelhantes, sendo as causas clínicas prevalentes nos seus atendimentos ${ }^{6}$. Contudo, leva-se em consideração que os traumas são atendidos também pelo SIATE. Porém o SAMU presta esta assistência devido à alta demanda de chamados ou, por muitas vezes, a população encaminhar o paciente direto para UPA do município e a ambulância do SAMU ser acionada pela central, 
a partir de protocolo para fazer a remoção para um hospital de referência.

Analisando ainda o maior número dos atendimentos, verifica-se que a faixa etária entre 19-59 anos teve destaque nos chamados, seguida respectivamente em ordem decrescente, das faixas etárias de 60 anos ou mais, 12 a 18 anos e 0 a 11 anos. Um estudo realizado no SAMU de Ipatinga (MG) no ano de 2018 apontou um perfil etário semelhante, sendo $662(64,90 \%)$ dos atendimentos pela população adulta de 19 e 59 anos $^{5}$.

Em divergência, outra pesquisa mostra que a faixa etária com prevalência nos atendimentos foi de 60-79 anos. Porém, para este caso leva-se em consideração que a análise do perfil dos atendimentos do município de Ijuí foi por uma Unidade de Suporte Avançado (USA), sendo os idosos uma população de maior complexidade devido às doenças crônicodegenerativas ${ }^{8}$.

Com relação ao destino dos atendimentos do SAMU de Colombo, observa-se que 13377 (63,75\%) da população tiveram como destino final a UPA do município. Ou seja, o fluxo condiz com a estruturação da Rede de Atenção às Urgências (RAU), que preconiza que a unidade deve funcionar de forma intermediária entre as Unidades Básicas de Saúde e os hospitais de referência9 ${ }^{9}$. Porém, podese considerar tal fato em virtude do município de Colombo possuir atualmente apenas um hospital (Hospital Maternidade), sendo a UPA a principal porta para os atendimentos de urgência e emergência.

Outro ponto observado foi um grande número de fichas de atendimento preenchidas de forma incompletas e/ou rasuradas - 398 (12,50\%), indicando falha no preenchimento pelos profissionais de saúde. Hoje, o registro de informações em prontuários ou fichas de atendimento é de extrema importância na área da saúde por ser considerado um documento legal, no qual se registra o quadro clínico e todos os cuidados prestados ao paciente, servindo como respaldo dos profissionais em questões éticas e jurídicas ${ }^{10}$. Este mesmo problema foi apresentado em outra análise de perfil do SAMU, onde houve um número significativo de fichas com letra ilegível ou com dados preenchidos de forma inadequada, limitando a abrangência do estudo?.

Em associação, a finalização dos encaminhamentos realizados pelas ambulâncias, 387 casos obtiveram liberação após o atendimento e 229 casos recusaram o atendimento, números estes consideráveis que descaracterizam um cenário de emergência. A procura pelo serviço móvel em situação de baixa gravidade ocorre em vários países do mundo e também em sistemas de saúde inadequadamente estruturados ${ }^{11}$. Este problema pode ser amenizado por meio de educação em saúde, para que a população utilize o serviço de forma adequada8.

A estatística do estudo poderá contribuir para que a gestão no município possa analisar o perfil dos atendimentos da comunidade e reavaliar a necessidade de planejamento e ações para qualificar a rede de assistência à saúde. E, para uma melhor rede assistencial, é preciso que o sistema de regulação atue de maneira efetiva e que as ações de saúde sejam sempre monitoradas através de indicadores, a fim de resolutividade no setor das urgências².

\section{CONCLUSÕES}

Os resultados demonstraram que o perfil dos atendimentos do SAMU no município de Colombo, no período de dezembro de 2016 a agosto de 2017 foi predominante no sexo masculino, faixa etária de 19 a 59 anos, ocorrências clínicas e encaminhados à UPA do município.

Os dados encontrados evidenciam que o SAMU teve alto índice de acionamento pela população logo que inaugurou sua base no município. Isso mostra o quanto a implantação deste serviço teve grande relevância para atender os usuários em possíveis situações de urgência e emergência. 
O estudo também mostra que a população adultojovem tem utilizado este serviço móvel com maior frequência em comparação à população idosa. Este fator pode estar associado à falta de prevenção desta faixa etária atualmente.

É necessário enfatizar a importância do registro correto nas fichas dos atendimentos realizados pelos profissionais atuantes neste serviço, pois além de ser procedimento padrão e obrigatório na rotina do atendimento, a falta ou incorreto preenchimento das informações pode ocasionar na descontinuação da assistência prestada. Tal fato foi o que limitou este estudo de incluir dados para uma melhor precisão.

A análise do perfil dos atendimentos pode auxiliar as autoridades e a gestão local a ter uma visão das principais características e demandas dos usuários, para que medidas de controle de promoção e prevenção na atenção primária sejam realizadas de forma efetiva e eficaz, a fim de atingir melhores resultados nos indicadores de saúde e utilização adequada do serviço de urgência e emergência.

Acredita-se que os resultados atingidos estimulem a realização de novos estudos na área de urgência e emergência com ênfase nos atendimentos prestados pelo serviço do SAMU.

\section{REFERÊNCIAS}

1. Soares MKP, Dantas RAN, Dantas DV, Nunes HMAN, Nascimento RA, Nascimento JCPN. Perfil dos usuários atendidos por um serviço pré-hospitalar móvel de urgência no Nordeste brasileiro. Revista de Pesquisa: Cuidado é Fundamental Online. [Internet] 2018 [acesso em $2020 \mathrm{fev}$ 17];10(2):503-509. Disponível em: http://www.seer.unirio.br/ index.php/cuidadofundamental/article/view/6111

2. Costa DF, Silva AKFD, Santana IMC. Ocorrências atendidas pelo suporte básico de vida no serviço de atendimento móvel de urgência. Revista Eletrônica Estácio Saúde. [Internet] 2015 [acesso em 2020 fev 17];4(2):139151. Disponível em: http://periodicos.estacio.br/index.php/ saudesantacatarina/article/view/1760
3. Gonsaga RAT, Brugugnolli ID, Zanutto TA, Gilioli JP, Silva LFC. Fraga GP. Características dos atendimentos realizados pelo Serviço de Atendimento Móvel de Urgência no município de Catanduva, estado de São Paulo, Brasil, 2006 a 2012. Epidemiol Serv Saúde. [Internet] 2013 [citado 2020 Fev 17]; 22(2):317-324. Disponível em: http://scielo. iec.gov.br/scielo.php?script=sci_arttext\&pid=S167949742013000200013\&lng=pt. http://dx.doi.org/10.5123/ S1679-49742013000200013.

4. Acosta AM, da Silva Lima MAD. Características de usuários frequentes de serviços de urgência: revisão integrativa. Revista Eletrônica de Enfermagem. Goiânia. [Internet] 2013 [acesso em 2020 fev 17];15(2):564-573. Disponível em: https://www.fen.ufg.br/revista/v15/n2/pdf/ v15n2a31.pdf

5. Andrade GGD, Alcântara LB, Moreira MAM, Fialho SDPG, Silva FM. Perfil dos Atendimentos realizados pelo Serviço de Atendimento Móvel de Urgência (SAMU) de Ipatinga (MG). Brazilian Journal of Surgery and Clinical Research - BJSCR. [Internet] 2018 [acesso em $2020 \mathrm{fev}$ 17];23(3):41-51. Disponível em: https://www.mastereditora. com.br/periodico/20180805_111853.pdf

6. Tibães HBB, Silva DM, Alves M, Mattos PCM, Brito MJM. Perfil de atendimento do Serviço de Atendimento Móvel de Urgência no Norte de Minas Gerais. Rev Fund Care Online. [Internet] 2018 [acesso em 2020 fev 17]; 10(3):675-682. Disponível em: https://pdfs.semanticscholar. org/5afd/f4307a7c53eadbeafcab85dad65bbbc4872b.pdf

7. Mendonça MFS, Silva APSC, Castro CCL. Análise espacial dos acidentes de trânsito urbano atendidos pelo Serviço de Atendimento Móvel de Urgência: um recorte no espaço e no tempo. Rev bras epidemiol. [Internet] 2017 [acesso em 2020 fev 17];20(4):727-741. Disponível em: http:// www.scielo.br/scielo.php?script=sci_arttext\&pid=S1415790X2017000400727\&lng=en.

8. Casagrande D, Stamm B, Leite MT. Perfil dos atendimentos realizados por uma Unidade de Suporte Avançado do Serviço de Atendimento Móvel de Urgência (SAMU) do Rio Grande do Sul. Scientia Medica (Porto Alegre). [Internet] 2013 [acesso em 2020 fev 17]; 23(3):149155. Disponível em: https://pdfs.semanticscholar.org/2fd6/ dd063b7eb14b89de0c1ff67cad4b2e8e205c.pdf

9. Dias JMC, de Lima MSM, Dantas RAN, Costa IKF, Leite JEL, Dantas DV. Perfil de atendimento do serviço préhospitalar móvel de urgência estadual. Cogitare Enferm. 
[Internet] 2016 [acesso em 2020 fev 17];21(1). Disponível em: https://revistas.ufpr.br/cogitare/article/view/42470

10. Barros BC, Lima KSB, Carmo AFS, Moura LA, Barros AG, Korinfisky JP. O instrumento de registro do SAMU: com a palavra dos profissionais de saúde. J Res.: Fundam Care Online. [Internet] 2014 [Acesso em $2020 \mathrm{fev}$ 17];6(2):618-626. Disponível em: https://www.redalyc.org/ pdf/5057/505750622017.pdf

11. O'Dwyer G, Konder MT, Reciputti LP, Macedo C, Lopes MGM. O processo de implantação do Serviço de Atendimento Móvel de Urgência no Brasil: estratégias de ação e dimensões estruturais. Cad Saúde Pública. [Internet]. 2017 [Acesso em 2020 fev 17]; 33: e00043716. Disponível em: http://www.scielo.br/scielo.php?script=sci_ abstract\&pid=S0102-311X2017000705010\&lng=en\&nrm=is o\&tlng=pt

12. Bugês AFC, Probst LF, Cavalcante DFB, Bulgareli JV. Estudo Quanti-Qualitativo do Fluxo de Regulação de Leitos Hospitalares no Paraná. Revista de Gestão em Sistemas de Saúde. [Internet] 2017 [Acesso em 2020 fev 17];6(3):265-275. Disponível em: http://www.revistargss.org.br/ojs/index.php/ rgss/article/view/317 